\title{
CONJECTURE IN ADDITIVE TWIN PRIMES NUMBERS THEORY
}

\author{
Ibrahima Gueye*
}

*Mathematician Amator

\section{Keywords: Additive twin primes numbers theory; conjecture, Goldbach's weak conjecture}

\begin{abstract}
For two millennia, the prime numbers have continued to fascinate mathematicians. Indeed, a conjecture which dates back to this period states that the number of twin primes is infinite. In 1949 Clement showed a theorem on twin primes. For the record, the theorem of Clement has quickly been known to be ineffective in

the development of twin primes because of the factorial. This is why I thought of using the additive theory of numbers to find pairs of twin primes from the first two pairs of twin primes. What I have formulated as a conjecture. In same time i present my idea about the solution of the Goldbach's weak conjecture.
\end{abstract}

\section{Introduction:}

Or (p, q) a pair of integers such that $\mathrm{p}$ and $\mathrm{q}$ are both prime and $\mathrm{p}<\mathrm{q}$. We say that $(\mathrm{p}, \mathrm{q})$ form a pair of twin primes if $q=p+2$.

The couple $(2,3)$ is the only pair of consecutive primes.

Omitting the pair $(2,3), 2$ is the smallest possible distance between two primes, twin primes are two and two consecutive odd numbers.

Any pair of twin primes (with the exception of the couple $(3,5))$ is of the form $(6 n-1,6 n+1)$ for some integer $\mathrm{n}$. Indeed, any set of three consecutive natural numbers has at least a multiple of 2 (possibly two) and one multiple of 3, these two are confused between multiple two twin primes.

It is possible to show that, for any integer, the pair $(\mathrm{m}, \mathrm{m}+2)$ consists of twin primes if and only if: $4[(\mathrm{~m}-1) !+1]+\mathrm{m}=0 \bmod \mathrm{m}(\mathrm{m}+2)$

This characterization of factorial and modular twin primes was discovered by P. A. Clement in 1949[4].

The series of reciprocals of twin primes converges to Brun's constant, unlike the series of reciprocals of prime numbers. This property was demonstrated by Viggo Brun in 1919 [6].

The twin prime conjecture states that there are infinitely many twin primes. In other words, there are infinitely many primes $\mathrm{p}$ such that $(\mathrm{p}+2)$ is also prime.

In 1940, Paul Erdős proved the existence of a constant $\mathrm{c}<1$ and infinitely many primes $\mathrm{p}$ such that: $p^{\prime}-p<\operatorname{cln}(p)$ where $p^{\prime}$ denotes the number immediately following the first $p$.

This result was improved several times, in 1986, Helmut Maier showed a constant $\mathrm{c}<0.25$ could be reached.

In 2003, Daniel Goldston and Cem Yildirim have shown that, for all $c>0$, there are infinitely many primes $p$ such that $p^{\prime}-p<\ln (p)$.

In 1966, Chen Jingrun demonstrated the existence of infinitely many primes $p$ such that $p+2$ is the product of at most two prime factors (such a number, product of at most two prime factors, 2 is said -almost-first).

His approach was that of the theory of the screen, he used to treat similarly the twin prime conjecture and Goldbach's Conjecture.

Between 2006 and 2009, Ben Green, Terence Tao and Tamar Ziegler gave in several of their articles that there is an infinity of integers $n$ and $m$ tells that: $n, m,(n+m+1)$ and $(n+2 m+2)$ are prime-numbers[5].

As for me I decided to go further and investigate the relationship there may be between the twin primes and additive number theory. In same time i present my idea about the solution of the Goldbach's weak conjecture.

In number theory, Goldbach's weak conjecture, also known as the odd Goldbach's conjecture or the 
problem of three prime numbers, says: odd numbers greater than or equal to 9 is sum of three odd primes.

A prime number can be used more than once in the same amount.

This conjecture is described as "weak" because the strong Goldbach conjecture on sums of two primes, if it were proven, would establish weak conjecture of Goldbach. Indeed, if every even number $\geq 6$ is the sum of two primes (necessarily odd), adding three to each even number $\geq 6$ will produce the odd numbers $\geq 9$.

The conjecture has not yet been demonstrated but we have partial proofs.

In 1923, Hardy and Littlewood showed that, assuming some true generalization of the Riemann hypothesis, Goldbach's conjecture is true low for all sufficiently large odd numbers.

In 1937, a Russian mathematician, Ivan Vinogradov, was able to eliminate dependence on the Riemann hypothesis and demonstrated directly that all sufficiently large odd numbers can be expressed as the sum of three primes. Of thresholds where this is true could be calculated, but they are still too high to allow verification by brute force calculation that all odd numbers less than the smallest of these thresholds verify the conjecture.

In 1997, Jean-Marc Deshouillers, Gove Effinger, Herman te Riele and Zinoviev Dmitry showed that the generalized Riemann hypothesis implies the conjecture of Goldbach low [2]. This result combines a general statement valid for numbers larger than 1020 with a systematic computer search for small cases.

In an article in January 31, 2012 entitled "Every odd number is Greater Than the Sum of 1 at most five primes", Terence Tao shows that every odd number can be written as a sum of at most five primes [1].

\section{Theorem 1: Theorem Wilson}

Statement: An integer $\mathrm{p}$ strictly greater than 1 , is a prime number if and only if divides $(\mathrm{p}-1) !+1$, that is to say if and only if:

$(\mathrm{p}-1) !+1=0(\bmod p)$

\section{Theorem 2: Theorem Clement}

Statement: For any integer, the pair $(\mathrm{m}, \mathrm{m}+2)$ consists of twin primes if and only if: $4[(\mathrm{~m}-1) !+\mathrm{m}]=0 \bmod \mathrm{m}(\mathrm{m}+2)$

\section{Theorem 3:}

Statement: There are infinitely many integers $n$ and $m$ tells that: $n, m,(n+m+1)$ and $(n+2 m+2)$ are prime numbers [2].

Conjecture 1: Goldbach's weak conjecture

Odd numbers greater than or equal to 9 is sum of three odd primes.

\section{Theorem 4:}

Every odd number greater than or equal to 3 can be written as a sum of at most five primes. $2 \mathrm{k}+1=\mathrm{a}+\mathrm{b}+\mathrm{c}+\mathrm{d}+\mathrm{e} ; \mathrm{n}$ is nonzero integer. $\mathrm{a} ; \mathrm{b} ; \mathrm{c} ; \mathrm{d}$ and e are primes or equal to 0 .

\section{Theorem 5:}

For a positive odd integer $\mathrm{p}$ and for any two distinct odd primes $\mathrm{p} 1$ and $\mathrm{p} 2$ with $\mathrm{p} 1+\mathrm{p} 2-\mathrm{p}=1$, then $(p-p 1) !(p-p 2) !=-1(\bmod p) \Leftrightarrow p$ is prime[3].

\section{Conjecture2:}

$\mathrm{J}$ be the set of twin primes.

$J$ ' is the set of integers $n$ and $m$ such that: $n, m,(n+2),(m+2),(n+m+1)$ and $(n+m+3)$ are prime numbers.

I guess that means all $\mathrm{J}^{\prime}$ is equal to the set $\mathrm{J}$ privated of $\{3 ; 7\}$.

NB: There are particular cases such as $n=m$.

We note $\mathrm{J}^{\prime}=\mathrm{J}-\{3 ; 7\}$ 


\section{Examples:}

$\{\mathrm{N}, \mathrm{m}\}=\{5,5\},\{\mathrm{n}, \mathrm{m}\}=\{5,11\},\{\mathrm{n}, \mathrm{m}\}=\{11,17\},\{\mathrm{n}, \mathrm{m}\}=\{11,29\}$

$\{\mathrm{N}, \mathrm{m}\}=\{17,41\},\{\mathrm{n}, \mathrm{m}\}=\{29,29\},\{\mathrm{n}, \mathrm{m}\}=\{29,41\}$

So far I have not found any contre-example.

\section{An idea about Goldbach's weak conjecture :}

We know that every odd number greater than 3 can be written as a sum of at most five primes.

$2 \mathrm{k}+1=\mathrm{a}+\mathrm{b}+\mathrm{c}+\mathrm{d}+\mathrm{e} ; \mathrm{n}$ is nonzero integer $\mathrm{a} ; \mathrm{b} ; \mathrm{c} ; \mathrm{d}$ and e are primes or equal to 0 .

$2 \mathrm{k}+1=(\mathrm{a}+\mathrm{b}+1)+(\mathrm{c}+\mathrm{d}+1)+(\mathrm{e}-2)$

There is an infinity of integers $n$ and $m$ tells that: $n, m,(n+m+1)$ and $(n+2 m+2)$ are prime numbers[5].

My idea is to study the primes $a ; b ; c ; d$ and e so that $(a+b+1)$ and $(c+d+1)$ are primes and e is the largest element of twin primes. This case is some complex.

$2 \mathrm{k}+1=(\mathrm{a}+\mathrm{b}+1)+(\mathrm{c}+\mathrm{d}-1)+\mathrm{e}$

Another simpler case and idea is to study primes $\mathrm{a} ; \mathrm{b} ; \mathrm{c} ; \mathrm{d}$ and e so that:

$(a+b+1)$ and $(c+d-1)$ are primes or

$(a+c+1)$ and $(b+d-1)$ are primes or

$(a+d+1)$ and $(c+b-1)$ are primes or

$(\mathrm{c}+\mathrm{b}+1)$ and $(\mathrm{a}+\mathrm{d}-1)$ are primes or

$(\mathrm{d}+\mathrm{b}+1)$ and $(\mathrm{c}+\mathrm{a}-1)$ are primes or

$(\mathrm{c}+\mathrm{d}+1)$ and $(\mathrm{a}+\mathrm{b}-1)$ are primes or

$(\mathrm{a}+\mathrm{b}+1)$ and $(\mathrm{c}+\mathrm{e}-1)$ are primes or $(\mathrm{a}+\mathrm{c}+1)$ and $(\mathrm{b}+\mathrm{e}-1)$ are primes or $(\mathrm{a}+\mathrm{e}+1)$ and $(\mathrm{c}+\mathrm{b}-1)$ are primes or $(\mathrm{c}+\mathrm{b}+1)$ and $(\mathrm{a}+\mathrm{e}-1)$ are primes or $(\mathrm{e}+\mathrm{b}+1)$ and $(\mathrm{c}+\mathrm{a}-1)$ are primes or $(\mathrm{c}+\mathrm{e}+1)$ and $(\mathrm{a}+\mathrm{b}-1)$ are primes or $(a+b+1)$ and $(e+d-1)$ are primes or $(a+d+1)$ and $(e+b-1)$ are primes or $(\mathrm{a}+\mathrm{e}+1)$ and $(\mathrm{b}+\mathrm{d}-1)$ are primes or $(\mathrm{d}+\mathrm{b}+1)$ and $(\mathrm{e}+\mathrm{a}-1)$ are primes or $(\mathrm{e}+\mathrm{b}+1)$ and $(\mathrm{a}+\mathrm{d}-1)$ are primes or $(\mathrm{d}+\mathrm{e}+1)$ and $(\mathrm{a}+\mathrm{b}-1)$ are primes or

$(\mathrm{a}+\mathrm{e}+1)$ and $(\mathrm{c}+\mathrm{d}-1)$ are primes or $(\mathrm{a}+\mathrm{c}+1)$ and $(\mathrm{e}+\mathrm{d}-1)$ are primes or $(\mathrm{a}+\mathrm{d}+1)$ and $(\mathrm{c}+\mathrm{e}-1)$ are primes or $(\mathrm{c}+\mathrm{e}+1)$ and $(\mathrm{a}+\mathrm{d}-1)$ are primes or $(\mathrm{d}+\mathrm{e}+1)$ and $(\mathrm{c}+\mathrm{a}-1)$ are primes or $(\mathrm{c}+\mathrm{d}+1)$ and $(\mathrm{a}+\mathrm{e}-1)$ are primes or

$(e+b+1)$ and $(c+d-1)$ are primes or $(\mathrm{c}+\mathrm{b}+1)$ and $(\mathrm{e}+\mathrm{d}-1)$ are primes or $(d+b+1)$ and $(c+e-1)$ are primes or $(\mathrm{e}+\mathrm{c}+1)$ and $(\mathrm{b}+\mathrm{d}-1)$ are primes or $(\mathrm{e}+\mathrm{d}+1)$ and $(\mathrm{c}+\mathrm{b}-1)$ are primes or $(\mathrm{c}+\mathrm{d}+1)$ and $(\mathrm{e}+\mathrm{b}-1)$ are primes or

For this case we have 30 combinaisons to study. If we proove that for every odd number at least one of these cases give a solution. 


\section{References:}

[1] Davide Castelvecchi, In Their Prime: Mathematicians Come Closer to Solving Goldbach's Weak Conjecture [archive], Scientific American, May 11, 2012

[2] Deshouillers, Effinger, Te Riele et Zinoviev, « A complete Vinogradov 3-primes theorem under the Riemann hypothesis ", Electronic Research Announcements of the American Mathematical Society, Vol 3, p. 99-104 (1997)

[3] K. Raja Rama Gandhi, Super six problems on primes, International Journal of Advancements in Research \& Technology, Volume 2, Issue1, January-2013 http://www.ijoart.org/docs/Supersix-problems-on-Primes.pdf

[4] PA Clement, Congruences for sets of premiums, American Mathematical Monthly 56 (1949), p. $23-25$

[5] Terence Tao, University of California, Los Angeles Mahler Lecture Series

[6] Viggo Brun, Series $1 / 5+1 / 7+1 / 11+1 / 13+1 / 17+1 / 19+1 / 29+1 / 31+1 / 41+1 / 43+1 / 59+$ $1 / 61+\ldots$ where denominators are "twin primes" is convergent or over, Bulletin of Mathematical Sciences 43 (1919), p. 100-104 and 124-128 